Supporting I nformation for

\title{
Novel Inhibitors of Human Histone Deacetylases: Design, Synthesis, Enzyme Inhibition, and Cancer Cell Growth Inhibition of SAHA-Based Non-hydroxamates
}

\author{
Takayoshi Suzuki, ${ }^{*}{ }^{\dagger}$ Yuki Nagano, ${ }^{\dagger}$ Akiyasu Kouketsu, ${ }^{\dagger}$ Azusa Matsuura,${ }^{\dagger}$ Sakiko Maruyama, ${ }^{\ddagger}$ Mineko \\ Kurotaki, ${ }^{\ddagger}$ Hidehiko Nakagawa, ${ }^{\dagger}$ and Naoki Miyata ${ }^{*}{ }^{\dagger}$ \\ ${ }^{\dagger}$ Graduate School of Pharmaceutical Sciences, Nagoya City University, 3-1 Tanabe-dori, Mizuho-ku, Nagoya, \\ Aichi 467-8603, Japan \\ ${ }^{\ddagger}$ Evaluation Group, Drug Research Department, R. \& D. Division, Pharmaceuticals Group, Nippon Kayaku \\ Co., Ltd., 31-12, Shimo 3-chome, Kita-ku, Tokyo 115-8588, Japan
}

\section{Elemental Analysis}
Compd
calcd
found
$4 \mathrm{C}_{13} \mathrm{H}_{19} \mathrm{~N}_{3} \mathrm{O}_{3}$
$\mathrm{C} ; 58.85, \mathrm{H} ; 7.22, \mathrm{~N} ; 15.84$
C; $59.03, \mathrm{H} ; 7.27, \mathrm{~N} ; 15.63$
$5 \quad \mathrm{C}_{13} \mathrm{H}_{20} \mathrm{~N}_{4} \mathrm{O}_{2}$
C; 59.07, H; 7.63, N; 21.20
C; 59.13, H; 7.56, N; 20.83
$6 \mathrm{C}_{13} \mathrm{H}_{20} \mathrm{~N}_{2} \mathrm{O}_{4} \mathrm{~S} \cdot 1 / 20 \mathrm{H}_{2} \mathrm{O}$
C; 51.83, H; 6.72, N; 9.30
C; 52.15, H; 6.88, N; 8.92
$7 \quad \mathrm{C}_{13} \mathrm{H}_{19} \mathrm{NOS}$
C; 65.78, H; 8.07, N; 5.90
C; 65.75, H; 8.08, N; 5.79
8a $\mathrm{C}_{15} \mathrm{H}_{21} \mathrm{NO}_{2} \mathrm{~S}$
C; 64.48, H; 7.58, N; 5.01
C; 64.19, H; 7.54, N; 4.66 
C; 67.05, H; 8.41, N; 5.69

$10 \mathrm{C}_{13} \mathrm{H}_{20} \mathrm{~N}_{2} \mathrm{O}_{3} \mathrm{~S}$

C; 54.91, H; 7.09, N; 9.85

C; 55.02, H; 7.19, N; 9.76

$11 \mathrm{C}_{14} \mathrm{H}_{21} \mathrm{NO}_{3} \mathrm{~S}$

C; 59.34, H; 7.47, N; 4.94

C; 59.06, H; 7.38, N; 5.21

$12 \mathrm{C}_{14} \mathrm{H}_{21} \mathrm{~N}_{3} \mathrm{O}_{2} \cdot$ TFA $\cdot 1 / 10 \mathrm{H}_{2} \mathrm{O}$

C; 50.68, H; 5.90, N; 11.08

C; 50.97, H; 5.88, N; 10.71

$13 \mathrm{C}_{14} \mathrm{H}_{20} \mathrm{~N}_{2} \mathrm{O}_{3}$

C; 63.62, H; 7.63, N; 10.60

C; 63.91, H; 7.72, N; 10.56

$14 \mathrm{C}_{14} \mathrm{H}_{20} \mathrm{~N}_{2} \mathrm{O}_{2} \mathrm{~S}$

C; 59.97, H; 7.19, N; 9.99

C; 59.90, H; 7.12, N; 10.09

$15 \mathrm{C}_{16} \mathrm{H}_{22} \mathrm{~N}_{2} \mathrm{O}_{3} \mathrm{~S}$

C; 59.60, H; 6.88, N; 8.69

C; 59.52, H; 6.93, N; 8.53

$16 \mathrm{C}_{15} \mathrm{H}_{20} \mathrm{~N}_{2} \mathrm{O} \cdot \mathrm{HCl} \cdot 1 / 8 \mathrm{H}_{2} \mathrm{O}$

C; 63.65, H; 7.57, N; 9.90

C; 63.63, H; 7.44, N; 9.91

$17 \mathrm{C}_{18} \mathrm{H}_{22} \mathrm{~N}_{2} \mathrm{O}$

C; 76.56, H; 7.85, N; 9.92

C; 76.29, H; 7.55, N; 9.79

$18 \mathrm{C}_{14} \mathrm{H}_{19} \mathrm{BrN}_{2} \mathrm{O}_{2}$

C; 51.39, H; 5.85, N; 8.56

C; 51.20, H; 5.72, N; 8.86

$19 \mathrm{C}_{14} \mathrm{H}_{21} \mathrm{NOS}$

C; 66.89, H; 8.42, N; 5.57

C; 66.90, H; 8.57, N; 5.25

$20 \mathrm{C}_{12} \mathrm{H}_{17} \mathrm{NOS}$

C; 64.53, H; 7.67, N; 6.27

C; 64.61, H; 7.78, N; 6.02

$21 \mathrm{C}_{11} \mathrm{H}_{15} \mathrm{NOS} \cdot 1 / 12 \mathrm{H}_{2} \mathrm{O}$

C; 62.67, H; 7.25, N; 6.64

C; 63.04, H; 7.15, N; 6.25

$23 \mathrm{C}_{13} \mathrm{H}_{19} \mathrm{NOS} \cdot 1 / 6 \mathrm{H}_{2} \mathrm{O}$

C; 64.96, H; 8.11, N; 5.83

C; 65.36, H; 8.02, N; 5.43 
$24 \mathrm{C}_{15} \mathrm{H}_{24} \mathrm{~N}_{2} \mathrm{OS}$

$25 \mathrm{C}_{19} \mathrm{H}_{23} \mathrm{NOS} \cdot 1 / 5 \mathrm{H}_{2} \mathrm{O}$

$26 \mathrm{C}_{19} \mathrm{H}_{23} \mathrm{NOS}$

$27 \mathrm{C}_{19} \mathrm{H}_{23} \mathrm{NO}_{2} \mathrm{~S}$

$28 \mathrm{C}_{19} \mathrm{H}_{23} \mathrm{NO}_{2} \mathrm{~S}$

$29 \mathrm{C}_{12} \mathrm{H}_{18} \mathrm{~N}_{2} \mathrm{OS}$

$30 \mathrm{C}_{16} \mathrm{H}_{20} \mathrm{~N}_{2} \mathrm{OS}$

$31 \mathrm{C}_{16} \mathrm{H}_{20} \mathrm{~N}_{2} \mathrm{OS}_{2} \cdot 1 / 10 \mathrm{H}_{2} \mathrm{O}$

$32 \mathrm{C}_{14} \mathrm{H}_{18} \mathrm{~N}_{2} \mathrm{OS}_{2}$

$33 \mathrm{C}_{15} \mathrm{H}_{24} \mathrm{~N}_{2} \mathrm{OS}$

$34 \mathrm{C}_{17} \mathrm{H}_{21} \mathrm{NOS}$

$35 \mathrm{C}_{15} \mathrm{H}_{19} \mathrm{NO}_{2} \mathrm{~S}$

$36 \mathrm{C}_{15} \mathrm{H}_{20} \mathrm{~N}_{2} \mathrm{OS}$
C; 64.24, H; 8.63, N; 9.99

C; 71.97, H; 7.44, N; 4.42

C; 72.18, H; 7.39, N; 4.64

C; 72.80, H; 7.40, N; 4.47

C; 72.65, H; 7.38, N; 4.77

C; 69.27, H; 7.04, N; 4.25

C; 69.26, H; 7.07, N; 3.89

C; 69.27, H; 7.04, N; 4.25

C; 69.63, H; 7.15, N; 3.95

C; 60.47, H; 7.61, N; 11.75

C; 60.64, H; 7.61, N; 11.39

C; 66.63, H; 6.99, N; 9.71

C; 66.66, H; 6.92, N; 9.54

C; 59.63, H; 6.32, N; 8.69

C; 60.01, H; 6.33, N; 8.31

C; 57.11, H; 6.16, N; 9.51

C; 56.82, H; 6.17, N; 9.31

C; 64.24, H; 8.63, N; 9.99

C; 64.22, H; 8.60, N; 9.67

$\mathrm{C} ; 71.04, \mathrm{H} ; 7.36, \mathrm{~N} ; 4.87$

C; 70.90, H; 7.40, N; 4.68

$\mathrm{C} ;$ 64.95, H; 6.96, N; 5.05

C; 65.00, H; 6.97, N; 4.77

C; 65.18, H; 7.29, N; 10.14

C; 65.32, H; 7.34, N; 9.88 

$37 \mathrm{C}_{26} \mathrm{H}_{36} \mathrm{~N}_{2} \mathrm{O}_{2} \mathrm{~S}_{2}$
C; 66.06, H; 7.68, N; 5.93
C; 65.94, H; 7.62, N; 5.66
$38 \mathrm{C}_{16} \mathrm{H}_{23} \mathrm{NO}_{2} \mathrm{~S}$
C; 65.49, H; 7.90, N; 4.77
C; 65.52, H; 8.02, N; 4.60
$39 \mathrm{C}_{17} \mathrm{H}_{25} \mathrm{NO}_{2} \mathrm{~S}$
C; 66.41, H; 8.20, N; 4.56
C; 66.55, H; 8.34, N; 4.43
$40 \mathrm{C}_{17} \mathrm{H}_{25} \mathrm{NO}_{2} \mathrm{~S}$
C; 66.41, H; 8.20, N; 4.56
C; 66.56, H; 8.22, N; 4.26
$41 \mathrm{C}_{18} \mathrm{H}_{27} \mathrm{NO}_{2} \mathrm{~S}$
C; 67.25, H; 8.47, N; 4.36
C; 67.08, H; 8.39, N; 3.98
$42 \mathrm{C}_{17} \mathrm{H}_{23} \mathrm{NO}_{2} \mathrm{~S}$
C; 66.85, H; 7.59, N; 4.59
C; 67.12, H; 7.74, N; 4.39
$43 \mathrm{C}_{19} \mathrm{H}_{27} \mathrm{NO}_{2} \mathrm{~S}$
C; 68.43, H; 8.16, N; 4.20
C; 68.82, H; 8.34, N; 3.86
$44 \mathrm{C}_{20} \mathrm{H}_{23} \mathrm{NO}_{2} \mathrm{~S}$
C; 70.35, H; 6.79, N; 4.10
C; 70.10, H; 6.81, N; 3.90
$45 \mathrm{C}_{20} \mathrm{H}_{22} \mathrm{~N}_{2} \mathrm{O}_{4} \mathrm{~S}$
C; 62.16, H; 5.74, N; 7.25
C; 62.04, H; 5.72, N; 7.05
$47 \mathrm{C}_{23} \mathrm{H}_{29} \mathrm{NO}_{2} \mathrm{~S}$
C; 72.02, H; 7.62, N; 3.65
C; 72.11, H; 7.66, N; 4.02
$49 \mathrm{C}_{16} \mathrm{H}_{24} \mathrm{~N}_{2} \mathrm{O}_{2} \mathrm{~S}$
C; 62.30, H; 7.84, N; 9.08
C; 62.11, H; 7.92, N; 8.81
$50 \mathrm{C}_{20} \mathrm{H}_{26} \mathrm{~N}_{2} \mathrm{O}_{2} \mathrm{~S}$
C; 67.01, H; 7.31, N; 7.81
C; 66.97, H; 7.39, N; 7.45
$51 \mathrm{C}_{20} \mathrm{H}_{26} \mathrm{~N}_{2} \mathrm{O}_{2} \mathrm{~S}_{2}$
C; 61.50, H; 6.71, N; 7.17
C; 61.62, H; 6.76, N; 7.38 
$52 \mathrm{C}_{18} \mathrm{H}_{24} \mathrm{~N}_{2} \mathrm{O}_{2} \mathrm{~S}_{2}$

$53 \mathrm{C}_{21} \mathrm{H}_{27} \mathrm{NO}_{2} \mathrm{~S}$

$54 \mathrm{C}_{19} \mathrm{H}_{25} \mathrm{NO}_{3} \mathrm{~S}$

$55 \mathrm{C}_{19} \mathrm{H}_{26} \mathrm{~N}_{2} \mathrm{O}_{2} \mathrm{~S}$
C; 59.31, H; 6.64, N; 7.68

C; 70.55, H; 7.61, N; 3.92

C; 65.68, H; 7.25, N; 4.03

C; 65.86, H; 7.56, N; 8.08
C; 59.13, H; 6.63, N; 7.46

C; 70.74, H; 7.71, N; 3.66

C; 65.96, H; 7.46, N; 3.81

C; 66.10, H; 7.74, N; 7.84 